\title{
Ribosome collisions in bacteria promote ribosome rescue by triggering mRNA cleavage by SmrB
}

Kazuki Saito ${ }^{1}$, Hanna Kratzat ${ }^{2}$, Annabelle Campbell ${ }^{1}$, Robert Buschauer ${ }^{2}$, A. Maxwell Burroughs ${ }^{3}$, Otto Berninghausen ${ }^{2}$, L. Aravind ${ }^{3}$, Roland Beckmann², Rachel Green ${ }^{1,4}$, Allen R. Buskirk ${ }^{1, *}$

${ }^{1}$ Department of Molecular Biology and Genetics and ${ }^{4}$ Howard Hughes Medical Institute, Johns Hopkins University School of Medicine, Baltimore, United States.

${ }^{2}$ Gene Center and Center for Integrated Protein Science Munich, Department of Biochemistry, University of Munich, Munich, Germany.

${ }^{3}$ Computational Biology Branch, National Center for Biotechnology Information, National Library of Medicine, National Institutes of Health, Bethesda, United States.

*To whom correspondence should be addressed: buskirk@jhmi.edu

\begin{abstract}
Ribosome rescue pathways recycle stalled ribosomes and target problematic mRNAs and aborted proteins for degradation. In bacteria, it remains unclear how rescue pathways distinguish ribosomes stalled in the middle of a transcript from actively translating ribosomes. In a genetic screen in $E$. coli, we discovered a novel rescue factor that has endonuclease activity. SmrB cleaves mRNAs upstream of stalled ribosomes, allowing the ribosome rescue factor tmRNA (which acts on truncated mRNAs) to rescue upstream ribosomes. SmrB is recruited by ribosome collisions; cryo-EM structures of collided disomes from E. coli and B. subtilis reveal a distinct and conserved arrangement of the individual ribosomes and the composite SmrB binding site. These findings reveal the underlying mechanism by which ribosome collisions trigger ribosome rescue in bacteria.
\end{abstract}




\section{Introduction}

Ribosomes often encounter obstacles that stop them in their tracks: the synthesis of roughly 1 out of every 250 proteins in E. coli ends in failure (1). Problems arise in many ways. Ribosomes arrest when they reach the 3 '-end of transcripts that lack a stop codon, for example, due to premature transcriptional termination or mRNA decay (2). Ribosomes also stall on intact messages when they encounter chemical damage in the mRNA $(2,3)$, codons that are decoded very slowly (4), or specific nascent peptide sequences that inhibit their own translation $(5,6)$. Although stalling is sometimes resolved productively, prolonged pauses often lead to aborted protein synthesis. Such translational failures are dangerous because they trap ribosomes in inactive complexes and produce incomplete proteins that can be toxic. To meet these challenges, bacteria have evolved ribosome rescue mechanisms that selectively recognize stalled ribosomes, rescue the ribosomal subunits, and target problematic mRNAs and nascent proteins for degradation (reviewed in (7)).

How do ribosome rescue factors in bacteria distinguish stalled ribosomes from actively translating ribosomes? There are two rescue pathways active in E. coli: the main one (mediated by tmRNA and its protein partner, $\mathrm{SmpB}$ ) and a backup pathway (mediated by ArfA) that is induced when tmRNA is overwhelmed (8). These pathways differ in fundamental ways: the tmRNA-SmpB complex enters ribosomes and encodes a short peptide tag to target the nascent peptide for proteolysis (9) whereas ArfA simply promotes peptidyl hydrolysis by recruiting the canonical release factor RF2 (10). Because both factors bind in the mRNA channel, their activity is inhibited by intact mRNA downstream of the stall site $(7,11-13)$. In cases where translation stalls on intact messages, the current model is that mRNA cleavage yields truncated mRNAs that become good substrates for rescue pathways. For example, ribosome stalling during termination in an inefficient context, Glu-Pro-stop (EP*), leads to mRNA cleavage in the ribosomal A site (14). mRNA cleavage is an attractive mechanism that could explain how rescue factors identify ribosomes that have irreversibly arrested. Yet the signal that triggers mRNA cleavage and the identity of the nucleases involved have remained elusive.

Recent work has revealed insights into how eukaryotic cells recognize and rescue stalled ribosomes. The E3 ubiquitin ligase Hel2 was identified in a genetic screen in yeast as a factor that promotes ribosome rescue by adding ubiquitin to specific ribosomal proteins (15). Hel2 is thought to recognize the interface between two small ribosomal subunits formed when an upstream ribosome collides into a stalled ribosome $(16,17)$. Collisions are the trigger that leads to ubiquitination of r-proteins and activation of downstream quality control pathways including subunit splitting, mRNA decay, and degradation of the nascent polypeptide (18). In the absence of ubiquitination systems and Hel2 homologs, however, it has been unclear whether similar mechanisms are at play in bacteria. 
Here, we report that the $E$. coli protein SmrB cleaves mRNA upstream of stalled ribosomes, promoting ribosome rescue. SmrB contains an SMR domain associated with nuclease activity (1921); SMR-domain proteins are broadly conserved in bacteria and eukaryotes as well as in a few archaeal lineages, one of the few rescue factors with a widespread presence across the three superkingdoms of life. We show that following SmrB cleavage at the $5^{\prime}$-boundary of the stalled ribosome, upstream ribosomes translate to the $3^{\prime}$-end of the upstream mRNA fragment and are rapidly rescued by tmRNA. We show that SmrB binds preferentially to collided ribosomes and only cleaves at stalling motifs where collisions occur, arguing that SmrB recognizes aberrant translation through recruitment to collided ribosomes. Moreover, we present cryo-EM structures of ribosome dimers stalled at specific stalling motifs in B. subtilis and E. coli, revealing a distinct and conserved architecture of disomes formed by collisions. In SmrB-bound disomes we define a composite binding site that explains how SmrB is specifically recruited to and activated by ribosome collisions. These findings establish that ribosome collisions are the trigger for ribosome rescue in $E$. coli through recruitment of the nuclease SmrB.

\section{Results}

\section{A genetic selection for novel rescue factors}

To identify novel factors that act early in the ribosome rescue pathway in $E$. coli, we performed a genetic selection similar to the one used previously to discover Hel2 in eukaryotes (15), searching for mutants that allow ribosomes to translate through a strong stalling motif and complete the translation of a downstream ORF. Our selection is based on a reporter construct encoding a fusion of NanoLuc upstream of the bleomycin resistance protein (Ble) that will confer growth on selective media (Fig 1A). Two control constructs are shown: one has a stop codon in between the genes and produces NanoLuc alone (Stop); the second is a direct fusion without any intervening stalling motif (Non-stall) and produces full-length fusion protein. In a third construct, we inserted the strong SecM arrest motif at the junction of NanoLuc and Ble (SecM); overexpression of reporters containing this stalling motif leads to mRNA cleavage and tagging of the nascent peptide by tmRNA (5).

We first confirmed that the SecM reporter undergoes stalling and ribosome rescue using antibodies against the Strep-tag at the N-terminus of the NanoLuc-Ble fusion protein. Although abundant full-length protein is visible in the Non-stall control, no full-length protein is detectable for the SecM reporter due to the strong arrest sequence (Fig 1B). To visualize the aborted protein product, we introduced the SecM reporter into cells lacking tmRNA (encoded by ssrA). Using the anti-Strep antibody, we observed truncated protein that is roughly the same size as NanoLuc produced from the Stop control (Fig 1B).

As another control, we developed a reporter with eight consecutive isoleucine residues at the fusion site; this reporter yields relatively little full-length protein detected by the anti-Strep 
A

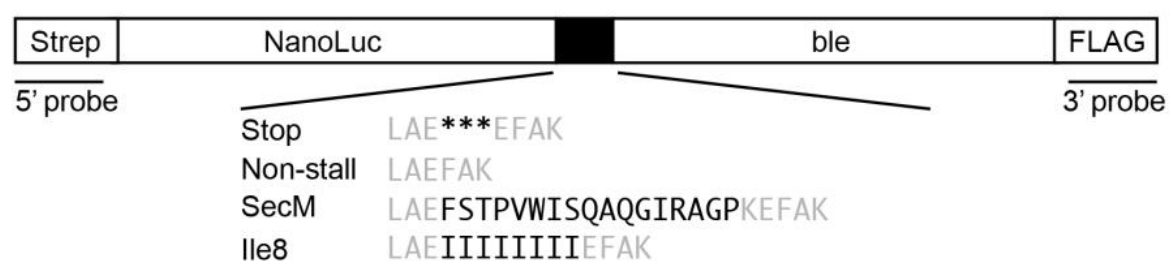
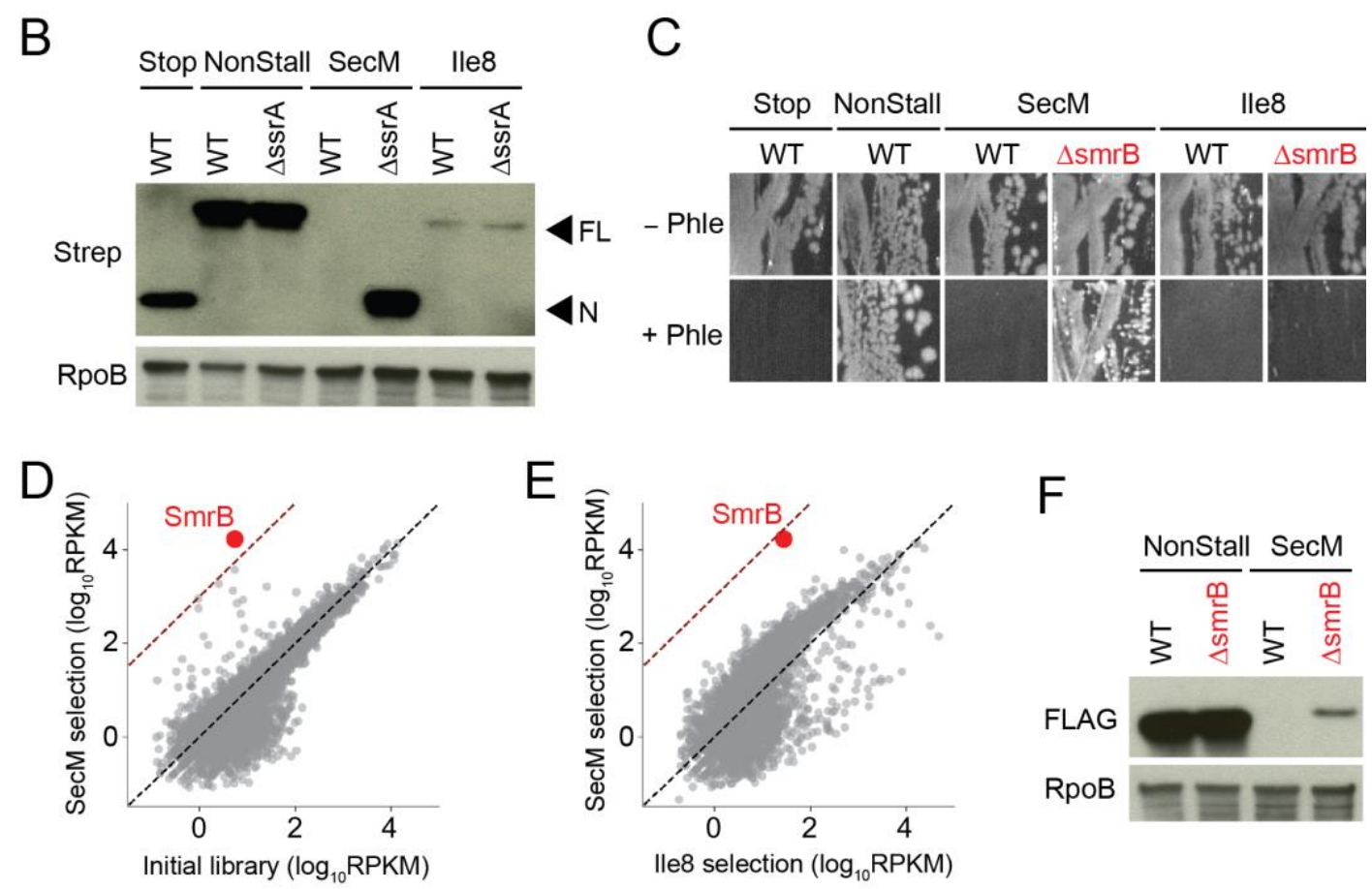

Figure 1. A genetic selection for novel rescue factors. (A) Reporters for studying ribosome rescue in $E$. coli. Between the NanoLuc gene and bleomycin resistance gene, we inserted either stop codons, no added sequence, the SecM stalling motif, or eight lle codons (black). (B) Reporter protein from wild-type and $\Delta$ ssrA strains was detected by antibodies against the $\mathrm{N}$ terminal Strep-tag. Arrows indicate the full-length fusion protein $(\mathrm{FL})$ and shorter NanoLuc protein $(\mathrm{N})$. The RpoB protein serves as a loading control. (C) Growth of wild-type and $\Delta \mathrm{smrB}$ strains expressing various reporters on media with or without $50 \mu \mathrm{g} / \mathrm{mL}$ phleomycin. (D) and (E) The results of TN-seq are shown as a scatter plot of mapped reads ( $\mathrm{rpkm}$ ) for each gene corresponding to the number of transposon insertions. The dashed line indicates 1000 -fold enrichment. (F) Full-length reporter protein was detected using antibodies against the FLAG-tag.

antibody, likely because the protein is misfolded and degraded (Ile8, Fig 1B). No truncated protein from the lle8 reporter was observed in the $\triangle$ ssrA strain. The relatively low levels of fulllength protein independent of ribosome stalling and rescue makes the lle8 reporter a useful control in our genetic selection, as discussed below.

Using the NanoLuc-Ble reporters, we found that expression of the full-length reporter protein from the Non-stall construct conveys resistance to $50 \mu \mathrm{g} / \mathrm{mL}$ phleomycin (an antibiotic structurally related to bleomycin) (Fig 1C). In contrast, cells expressing NanoLuc alone from the Stop construct are sensitive to this concentration of phleomycin, as expected. Next, we tested the SecM and lle8 constructs and observed that wild-type cells expressing these reporters are also phleomycin sensitive, in line with the finding that the full-length protein is expressed at very 
low levels from these constructs. These results establish parameters for the screen to identify gene deletions that increase the level of full-length reporter protein.

To perform the genetic selection we adopted a Tn-seq approach $(22,23)$, creating a knockout library of about 5 million colonies through random insertion of $\operatorname{Tn} 5$ transposase into $E$. coli K12 MG1655. We transformed this library with a plasmid expressing the SecM or the lle8 reporter and plated the transformants on media containing $50 \mu \mathrm{g} / \mathrm{mL}$ phleomycin. After harvesting phleomycin-resistant cells, we counted the number of transposon insertions per gene (normalized by length) in units of reads per kilobase per million mapped reads (rpkm) (Fig 1D). Compared to the initial library, 29 genes in the SecM reporter strain and 109 genes in the lle8 reporter strain exhibited a more than 10 -fold enrichment in transposon insertions. Many of these genes are false positives relevant to phleomycin toxicity. By comparing the results of the SecM selection with the lle8 selection, we can remove false positives from consideration, focusing instead on genes that are selectively enriched in the SecM selection because they affect ribosome stalling and rescue (Fig 1E).

We found a single gene, $s m r B$, where transposon insertions were strongly enriched in the SecM reporter strain compared to the lle8 reporter strain ( $600-$ fold, Fig 1E). To confirm this phenotype, we constructed a clean $s m r B$ deletion strain and found that $\Delta s m r B$ cells expressing the SecM reporter are resistant to phleomycin whereas cells expressing the lle8 reporter remain sensitive (Fig 1C). Furthermore, using the anti-FLAG antibody, we observe that deletion of $s m r B$ yields increased levels of full-length NanoLuc-Ble protein from the SecM reporter (Fig 1F) and from similar reporters with other stalling motifs, such as 12 rare Arg codons or the Glu-Pro-stop motif (EP*) (Fig S1). In contrast, loss of $s m r B$ has no effect on expression of the Non-stall reporter.

\section{SmrB is a conserved nuclease involved in ribosome rescue}

The $E$. coli SmrB protein is 183 amino acids long and contains a domain of the Small MutS Related (SMR) superfamily first proposed to act as a DNase in MutS-like proteins in bacteria and plants. However, more recent studies have shown that SMR domains possess endonucleolytic RNase activity $(19,24,25)$. Indeed, we recently identified an SMR protein, Cue2, as the endonuclease that cleaves mRNA upstream of stalled ribosomes in yeast (20). A systematic analysis of the SMR domains across the tree of life revealed independent fusions to a diverse array of RNA-binding domains supporting the hypothesis that it primarily operates on RNA (21).

To better understand the evolutionary trajectories of the SMR proteins, we performed phyletic pattern (Fig 2A) and phylogenetic analyses (Fig S2). We find that SMR domains are broadly conserved in bacteria, though notably underrepresented in the PVC group and the actinobacteria (Fig 2A). They are found across all sampled eukaryotic lineages, but are relatively uncommon in Archaea, where they are found mainly in Asgardarchaeota and Thermoplasmatota. 
bioRxiv preprint doi: https://doi.org/10.1101/2021.08.16.456513; this version posted August 16, 2021. The copyright holder for this preprint (which was not certified by peer review) is the author/funder, who has granted bioRxiv a license to display the preprint in perpetuity. It is made available under aCC-BY-NC-ND 4.0 International license.

A

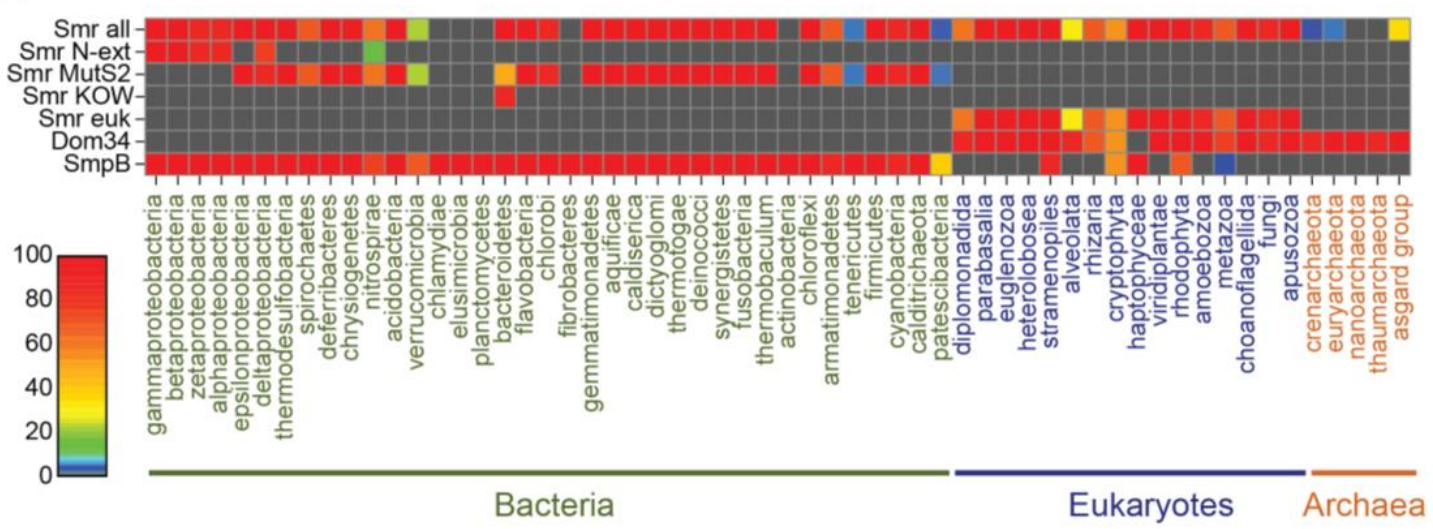

B

$\begin{array}{cccc}\text { N-ext Smr } & \begin{array}{c}\text { core/ } \\ \text { lever }\end{array} & \text { clamp } & \begin{array}{c}\text { MutS-like } \\ \text { ABC ATPase }\end{array} \\ \text { E. coli } & \text { BOW Smr } & \text { KOW Smr }\end{array}$

C

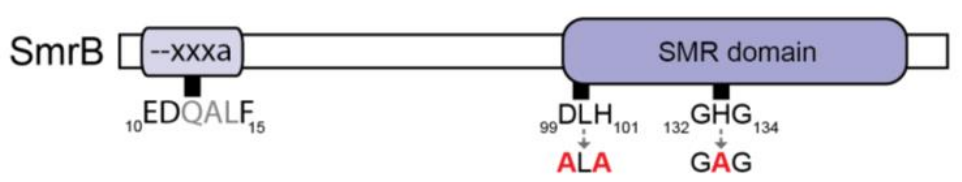

D

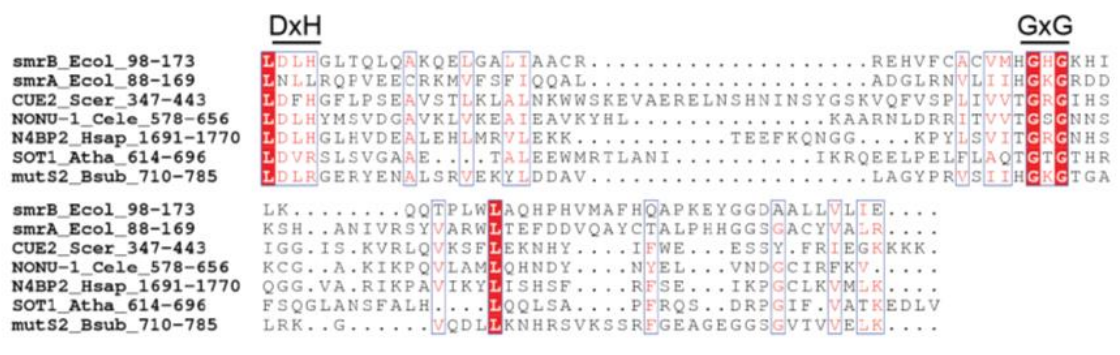

E
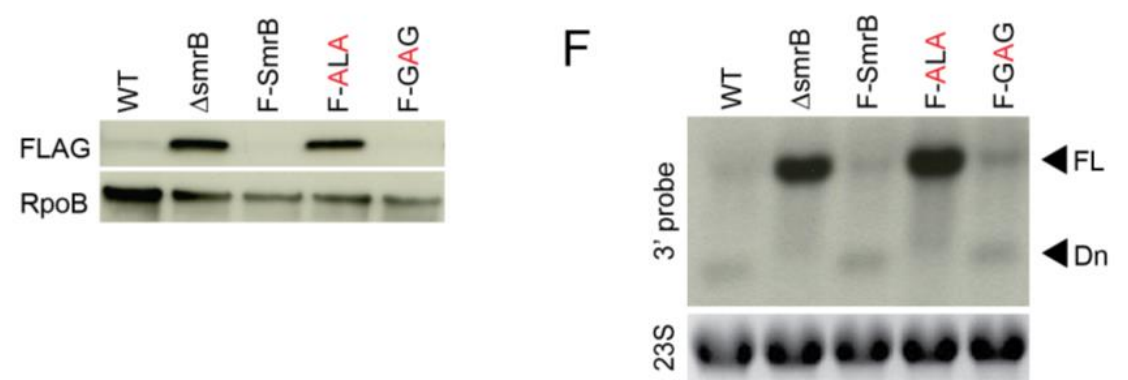

Figure 2. SMR-domain proteins are conserved RNA nucleases. (A) Heat map demonstrating the conservation and distribution of SMRdomain proteins and other related translational quality control factors. Smr-all includes all types of SMR-domain proteins; Smr-euk includes only the eukaryotic branch. (B) Domain organization of three representative bacterial proteins containing an SMR domain. (C) Domain organization of E. coli SmrB. Mutations at the two conserved DxH and GxG motifs are indicated by red bold letters. (D) Sequence alignment of SMR domains of representative proteins. Identical residues are shown in white with a red background; conserved residues are shown in red. The identity of each sequence is represented by the gene name, species name, and numbers indicating the beginning and the end of the residues used for the alignment. Ecol, Escherichia coli; Scer, Saccharomyces cerevisiae; Cele, Caenorhabditis elegans; Hsap, Homo sapiens; Atha, Arabidopsis thaliana; Bsub, Bacillus subtilis. (E) Full-length SecM reporter protein was detected with antibodies against the FLAG-tag. The RpoB protein serves as a loading control. F-SmrB, F-ALA, and F-GAG indicate endogenously FLAG-tagged SmrB and its variants. (F) The SecM reporter mRNA was detected on northern blots using a probe which anneals to the $3^{\prime}$-end of the reporter. Ethidium bromide staining of $23 \mathrm{~S}$ rRNA serves as a loading control. $\mathrm{FL}=$ full-length and $\mathrm{Dn}=$ the downstream mRNA fragment. 
These data suggest that the SMR domain might have entered the eukaryotic stem lineage at some point from a bacterial source.

The bacterial SMR domains cluster into three major clades. E. coli SmrB together with other proteobacterial versions form the first of these clades (Fig 2B) typified by a characteristic extension N-terminal to the SMR domain. This extension contains a predicted helix (residues 920) with a strongly conserved "--xxxa" motif (two negatively charged residues followed by three variable and one aromatic residue; $\mathrm{E}_{10} \mathrm{DQALF}_{15}$ in E. coli SmrB; Fig $2 \mathrm{C}$ and Fig S3) followed by a poorly conserved, largely unstructured segment. In gammaproteobacteria, like $E$. coli, a duplication led to two copies of these SMR proteins per genome; one with active site residues conserved $(\mathrm{SmrB})$ and the other predicted to be enzymatically inactive (SmrA). The second clade contains the most common bacterial version typified by the $B$. subtilis MutS2 protein. From N- to C-terminus, these proteins contain the core/lever and clamp domains, the MutS DNA mismatch repair protein-type P-loop ABC ATPase domain, as well as an additional KOW domain with a SH3like fold and a C-terminal SMR domain (Fig 2B). Notably, the MutS2 proteins lack the mismatch recognition and connector domains typical of the canonical MutS protein involved in mismatch repair. We identified several independent occasions where the predicted active site residues have been lost in this clade. The third clade is the smallest, restricted to the Bacteroidetes lineage, with an N-terminal KOW domain and C-terminal SMR domain.

An alignment of the SMR domain (residues 98-173) reveals that SmrB contains the conserved residues associated with endonuclease and RNA-binding activity $(21,26)$ : residues $\mathrm{D}_{99} \mathrm{LH}_{101}$ correspond to the DxH motif implicated in catalysis and residues $\mathrm{G}_{132} \mathrm{HG}_{134}$ with the $\mathrm{GxG}$ motif marking the loop predicted to interact with RNA substrates (Fig 2D). We generated E. coli strains where the DxH and GxG motifs were mutated to ALA and GAG, respectively (Fig 2C), at the endogenous $s m r B$ locus tagged with an N-terminal FLAG epitope. The FLAG tag does not inhibit SmrB activity; like the wild-type, little or no full-length reporter protein is detectable in this strain (Fig 2E). Importantly, we observe that the ALA mutation increased full-length protein to a similar extent as deletion of $s m r B$, whereas the GAG mutation had no discernable effect in vivo activity. These results suggest that the DxH motif is critical for SmrB activity while the central residue in the GxG motif is not required, consistent with its lower conservation. We note that loss of a second SMR-domain protein encoded in the $E$. coli genome, SmrA, which lacks the DxH motif did not affect expression of the stalling reporter (Fig S4).

To observe more directly the activity of SmrB on the reporter mRNA in vivo, we performed northern blots with a probe binding to the 3 '-end of the reporter construct (Fig 2F). In the wildtype strain, the full-length reporter mRNA is barely detectable while the strongest signal comes from a shorter mRNA fragment whose size suggests that cleavage is occurring somewhere near the SecM motif. In the $\Delta$ smrB strain, the downstream fragment disappears and the levels of the full-length mRNA are dramatically higher. As expected, the strains with FLAG-tagged SmrB and the GAG mutant show robust levels of RNA cleavage, whereas the ALA mutant strain shows high 
levels of reporter mRNA. These results confirm that SmrB cleaves the reporter mRNA in vivo and reveal that SmrB cleavage is the dominant pathway that targets the reporter mRNA for degradation.

\section{Reporter mRNAs decayed via multiple pathways}

We next asked whether we could detect the upstream fragment using a probe against the 5 -end of the reporter mRNA. We began by comparing the mRNA levels of the SecM-short reporter and the EP* reporter in the wild-type strain versus strains lacking either tmRNA or SmrB or both. Whereas we see little reporter RNA in the wild-type strain (since it is cleaved and degraded), the $5^{\prime}$-probe reveals the upstream fragment from both reporters in the $\Delta$ ssrA strain (Fig 3A), consistent with prior reports that the loss of tmRNA stabilizes the upstream fragment $(5,14)$. As expected, there is no detectable upstream fragment in the $\Delta s m r B$ strain. Surprisingly, however, the upstream fragment is present for both reporters in the $\Delta \operatorname{ssr} A \Delta \operatorname{smr} B$ strain $(\Delta \Delta)$; this unexpected result reveals that this truncated mRNA can be produced by one or more mechanisms that are independent of SmrB. It is unlikely that other endonucleases are responsible, given that the downstream fragment detected by the 3 '-probe disappears in strains lacking SmrB (Fig 3A). We speculate that in the absence of SmrB, the upstream fragment is generated by exonucleolytic decay of the mRNA back to the stalled ribosome; $E$. coli has three processive $3^{\prime}-5^{\prime}$ exonucleases implicated in mRNA decay but lacks 5'-3' exonucleases (27).

\section{SmrB cleaves $m R N A$ at the 5'-boundary of stalled ribosomes}

The reporter mRNA is degraded by at least two mechanisms, one that depends on SmrB and another that likely involves exonucleases. To better characterize these pathways, we used RACE to identify the 5'- and 3'-ends of the mRNA fragments produced by these decay events coupled to ribosome stalling (Fig 3B). 5'-RACE experiments reveal the 5 '-end of the downstream fragment which is generated solely by endonucleolytic cleavage by SmrB: there is a sharp peak $11 \mathrm{nt}$ upstream of the SecM stall site (Fig $3 \mathrm{C}$ ). This peak is also evident in the $\Delta$ ssrA strain, but disappears in the $\Delta \mathrm{smrB}$ and the $\Delta \mathrm{smrB} \Delta \mathrm{ssrA}$ knockout strains. These data are consistent with the northern blots showing that the downstream fragment is not detectable in strains lacking SmrB (Fig 3A, 3'-probe). We see very similar results from the EP* reporter where the $5^{\prime}$-end of the downstream fragment is $10 \mathrm{nt}$ upstream of the stop codon in the A site of the ribosome (Fig 3D). Based on ribosome footprinting experiments (28), we know that the 5 '-boundary of the ribosome is roughly $12 \mathrm{nt}$ upstream of the A-site codon, suggesting that SmrB cleaves mRNA at the site where it exits the ribosome.

We also used 3'-RACE to determine the 3 '-end of the upstream mRNA fragment (Fig 3B). The data from these experiments are more complex because multiple pathways generate truncated mRNAs near the stall site. For the SecM reporter, in the wild-type and $\Delta$ ssrA strains, the strongest peak in the $3^{\prime}$-RACE data is $11 \mathrm{nt}$ upstream of the stall site (Fig 3E); in the absence 
A

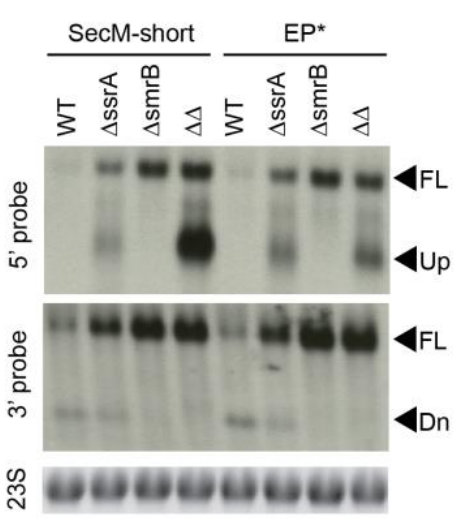

B

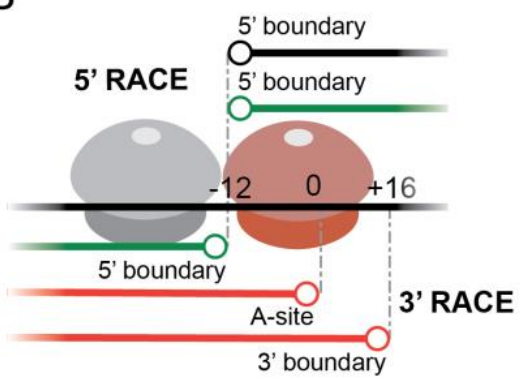

C

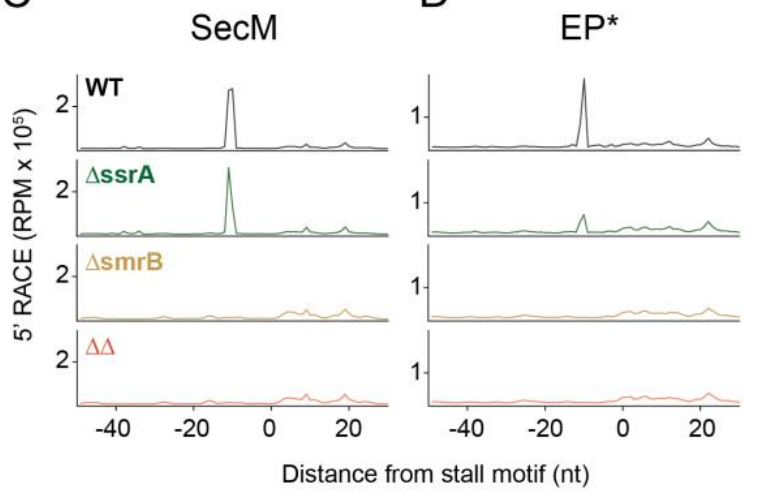

E

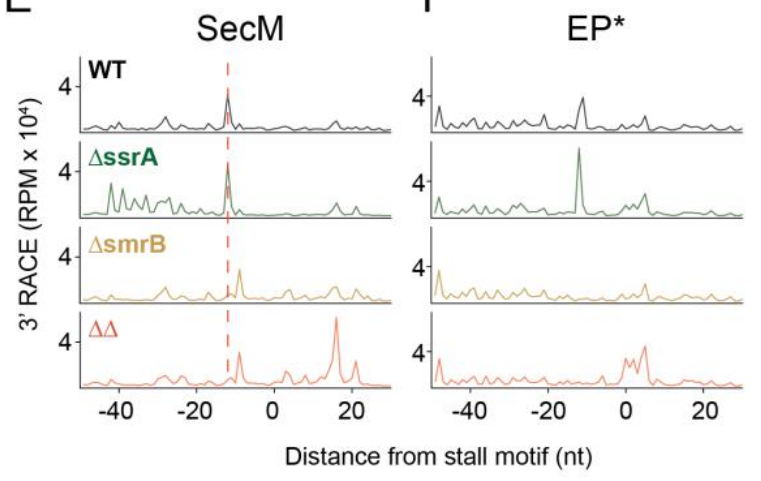

G
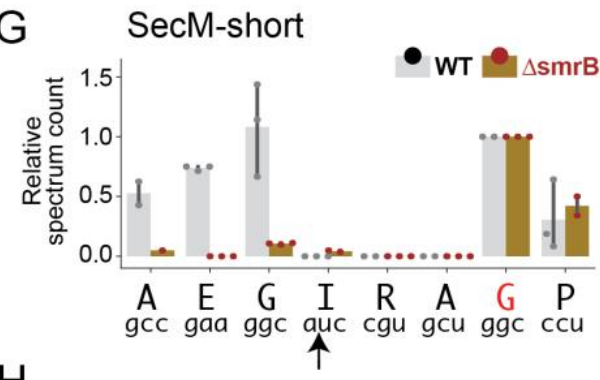

$\mathrm{H}$

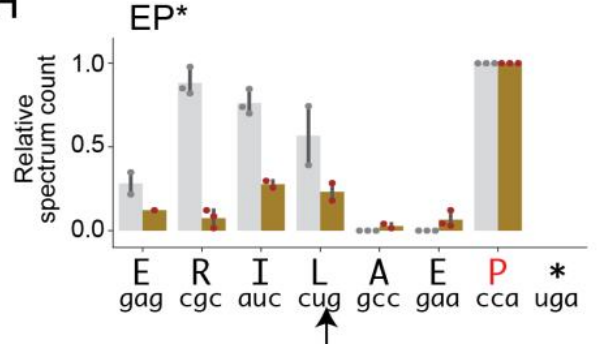

I

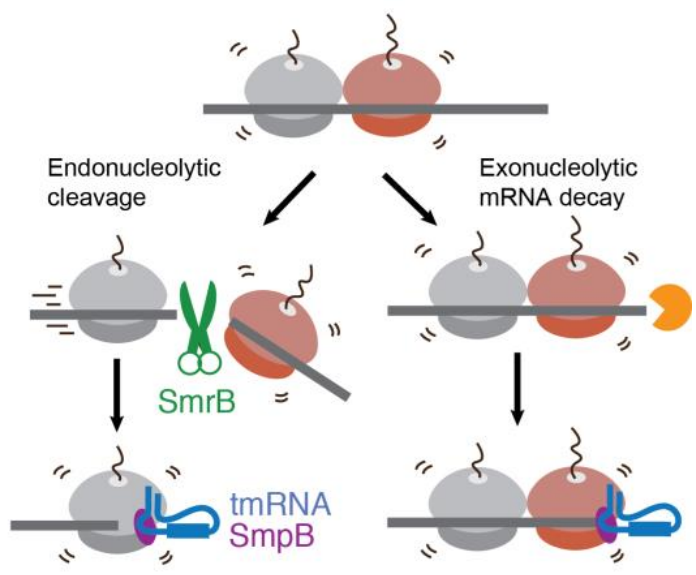

Figure 3. SmrB cleaves at the $5^{\prime}$ boundary of stalled ribosomes. (A) Northern blots of reporter mRNA using the $5^{\prime}$-probe and the $3^{\prime}$-probe. Arrows indicate the full-length (FL) or truncated RNAs (upstream or downstream fragments). Ethidium bromide staining of 235 rRNA serves as a loading control. (B) Schematic representation of RNA fragments observed in the RACE data. (C) and (D) The results of 5' RACE showing the $5^{\prime}$-ends of downstream fragments in reads per million on the reporter sequence. The first $\mathrm{nt}$ in the $\mathrm{A}$ site codon in the stall motif is designated as zero. (E) and (F) The results of $3^{\prime}$ RACE showing the $3^{\prime}$-ends of upstream fragments in reads per million on the reporter sequence. The first $\mathrm{nt}$ in the A site codon in the stall motif is designated as zero. (G) and (H) tmRNA tagging near the stall motif in wild-type and $\triangle$ smrB strains. The tagging site is the residue immediately preceding the tmRNA tag in peptide sequences detected by targeted LC-MS-MS. The red letter indicates the residue encoded by the P site codon at the stall site. The arrow indicates the SmrB cleavage site demonstrated by 5' RACE. The relative spectrum count is shown, normalized by the count at the stall site where tmRNA tagging was expected to occur in both the wildtype and $\Delta s m r B$ strains. (I) A model for mRNA processing during ribosome rescue. In the endonucleolytic cleavage pathway, SmrB cleaves the mRNA at the $5^{\prime}$ boundary of stalled ribosomes, at the interface of ribosome collisions. Upstream ribosomes then resume translation, reaching the $3^{\prime}$-end of the cleaved mRNA, and are quickly rescued by tmRNA-SmpB. In the secondary pathway, $3^{\prime}$-to-5' exonucleases degrade mRNA until they hit the $3^{\prime}$ boundary of the stalled ribosome, after which the stalled ribosome is eventually rescued by tmRNA-SmpB. 
of SmrB, this peak disappears. This same phenomenon was also observed in the EP* reporter (Fig $3 F$ ). For both reporters, these positions correspond perfectly with the site of cleavage identified on the downstream fragment by $5^{\prime}-\mathrm{RACE}$, suggesting that these upstream fragments are derived from SmrB cleavage at the $5^{\prime}$ boundary of stalled ribosomes.

In strains lacking both SmrB and tmRNA $(\Delta \Delta)$ the $3^{\prime}$-RACE data provide additional information about other pathways that act on the upstream mRNA fragment. In the absence of both factors, the strongest 3'-RACE signal for the SecM reporter is $16 \mathrm{nt}$ downstream of the first nt in the A site codon, roughly corresponding to the $3^{\prime}$-boundary of the ribosome stalled at the SecM motif (Fig 3E), likely the products of exonucleolytic decay. In contrast, the strongest 3'RACE signal from the double knockout strain expressing the EP* reporter is at the A-site codon (Fig 3F). These results are broadly consistent with previous reports of mRNA cleavage at both the 5'- and 3'-boundaries of ribosomes stalled on SecM (5) and of A-site cleavage within ribosomes stalled during termination at EP* (14). Taken together, the 5'- and 3'-RACE data on these stalling reporter mRNAs reveal that SmrB cleaves at the $5^{\prime}$-boundary of stalled ribosomes and that in the absence of SmrB, other pathways lead to mRNA decay up to the A-site codon or the 3'-boundary of the lead stalled ribosome.

\section{SmrB cleavage liberates stacked ribosomes upstream of stalled ribosomes}

Our analyses of the reporter mRNA indicate that SmrB cleaves upstream of stalled ribosomes forming an upstream fragment whose decay is promoted by tmRNA. To ask which ribosome complexes are rescued by tmRNA, we determined where the tmRNA tag is added on both the short-SecM and EP* reporters. We immunoprecipitated reporter protein (using the $\mathrm{N}$ terminal Strep-tag) from both the wild-type and $\triangle$ smrB strains containing a tmRNA variant that tags the reporter protein with a ClpXP-resistant AANDENYALDD sequence (4).

We determined the sites of tmRNA tagging by digesting the immunoprecipitated reporter protein with lysyl endopeptidase and subjecting the resulting peptides to LC-MS-MS. For the SecM reporter, where ribosomes stall with the second Gly codon in GIRAGP in the P site (29), there is strong signal from the peptide produced when the tmRNA tag is added at the second Gly residue (Fig 3G), as previously reported (5). Importantly, robust tagging at this site is observed in both the wild-type and $\Delta s m r B$ strains, suggesting that SmrB cleavage is not essential for tagging at the SecM stall site. We also observe strong tagging four residues upstream (at the first Gly) in the wild-type strain (Fig 3G). This result correlates precisely with the SmrB cleavage site determined by RACE; tagging at this site is dramatically reduced in the $\triangle$ smrB strain. Likewise, consistent with previous studies (6), we observe tmRNA tagging of the EP* reporter protein at the C-terminal Pro residue in both the wild-type and $\Delta$ smrB strains (Fig $3 \mathrm{H}$ ). We also observe tagging at the residues upstream of the stalling site at positions where mRNA is cleaved by SmrB in the wild-type strain; this signal is diminished in the $\Delta \mathrm{smrB}$ strain. 
Taken together, the RACE and tmRNA tagging results lead us to propose the following model for ribosome rescue within ORFs in E. coli (Fig 3I). Ribosome stalling leads to endonucleolytic cleavage by SmrB at the 5'-boundary of the first, stalled ribosome (red). No longer impeded by the ribosome trapped on the stalling motif, upstream ribosomes (grey) translate to the end of the upstream mRNA fragment and arrest at the 3 '-end generated by SmrB cleavage. The tmRNA-SmpB complex then rescues and releases these ribosomes, consistent with its well-characterized preference for truncated mRNAs. This model highlights how SmrB cleavage and tmRNA activity rapidly clear upstream ribosomes from the message and target it for decay. Consistent with previous studies, tmRNA also releases the initial stalled ribosome (red), tagging the nascent peptide right at the stall site. Decay of the reporter mRNA back to the 3'-boundary of the ribosome (in the case of SecM) or the A-site codon (in the case of EP*) by exonucleases likely allows tmRNA to gain access to these stalled ribosomes $(5,14,30)$.

\section{SmrB preferentially binds collided ribosomes}

We next asked how SmrB selectively recognizes stalled ribosomes. Although ribosome collisions have not been implicated in bacterial ribosome rescue, our data suggest that SmrB, like Cue2, recognizes collided ribosomes. First, as shown above, 5'-RACE of the downstream fragment in the SecM reporter reveals that SmrB cleavage occurs precisely at the 5 '-boundary of the SecMstalled ribosome; additional peaks are seen (with lower intensity) further upstream of the stall site that cluster in sets centered roughly $25 \mathrm{nt}$ apart, the length of a ribosome footprint (Fig 4A). Second, ribosome profiling data from the $\Delta s s r A \Delta s m r B$ strain expressing a related reporter reveal a strong peak of ribosome density at the SecM stall site, as expected, as well as sets of peaks roughly $25 \mathrm{nt}$ apart extending upstream (Fig 4A). The 5'-boundaries of the SecM stalled ribosome and the first three stacked ribosomes align well with the sites of SmrB cleavage seen in the RACE data. These findings suggest that SmrB cleavage happens in the context of ribosome collisions, arguing that collisions may serve as a signal for SmrB recruitment or activation.

Our work so far has focused on strong stalling motifs in reporter genes. To ask what effects collisions have on SmrB binding to ribosomes globally, we induced collisions throughout the transcriptome using the antibiotic mupirocin (MPC) which inhibits isoleucine tRNA synthetase and globally slows down the rate of decoding of lle codons $(28,31)$. In untreated cells, FLAG-SmrB is distributed broadly across the sucrose gradient, extending from the subunit fractions to the polysome fractions (Fig 4B), arguing that most SmrB is ribosome-bound. After inducing collisions for 5 min with $50 \mu \mathrm{g} / \mathrm{mL}$ MPC, we observed that FLAG-SmrB moves deeper into the polysome fraction, consistent with preferential binding to collided ribosomes on messages that are heavily translated (Fig 4B).

Further, we treated cell lysates with RNase A to generate nuclease-resistant disomes, a hallmark of ribosome collisions (17). As expected, RNase A treatment collapses polysomes to yield a strong monosome peak and a small nuclease-resistant disome peak (Fig 4C). The disome 
bioRxiv preprint doi: https://doi.org/10.1101/2021.08.16.456513; this version posted August 16, 2021. The copyright holder for this preprint (which was not certified by peer review) is the author/funder, who has granted bioRxiv a license to display the preprint in perpetuity. It is made available under aCC-BY-NC-ND 4.0 International license.

A
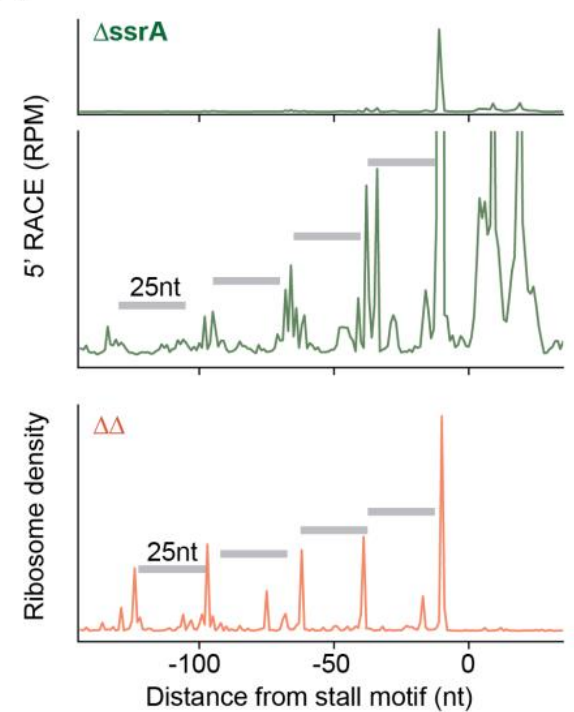

D

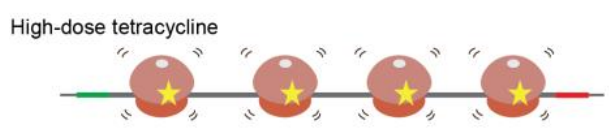

Low-dose tetracycline

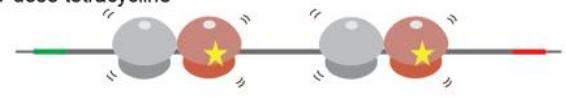

Sub-lethal tetracycline

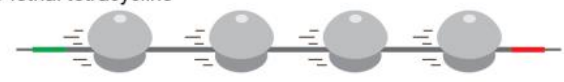

$\mathrm{F}$

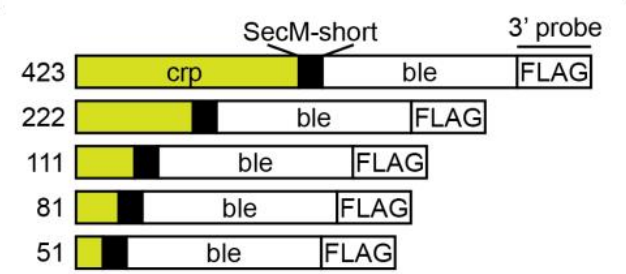

B

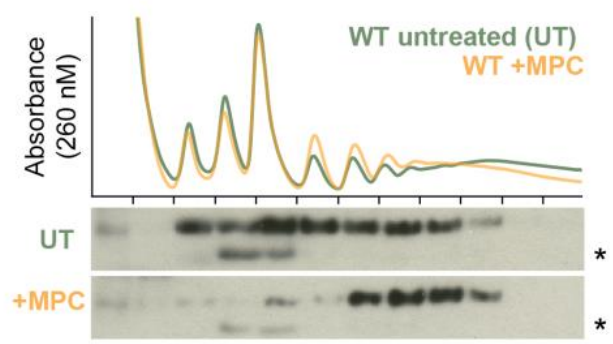

C

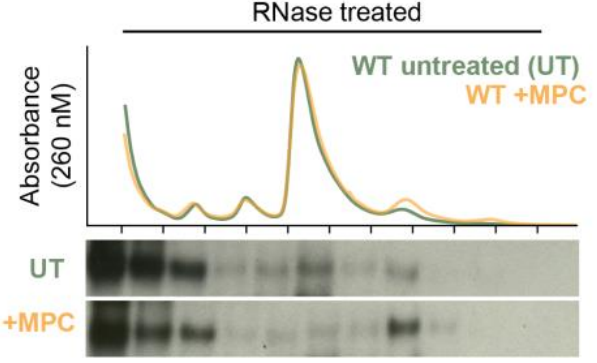

$\mathrm{E}$

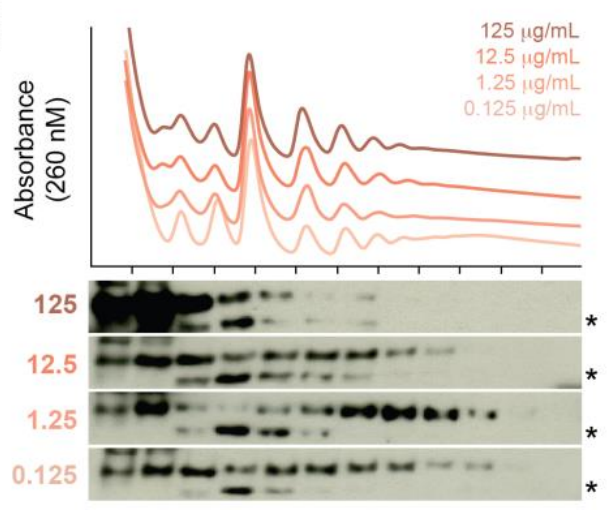

G

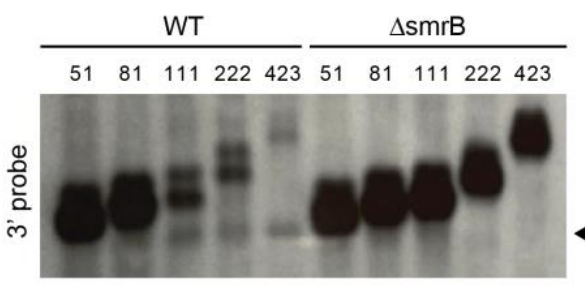

ஜ

Figure 4. SmrB preferentially binds and cleaves upstream of collided ribosomes. (A) Top: 5'-RACE data reveal the SmrB cleavage sites as in Fig 4C, zoomed in to show smaller peaks upstream. Bottom: ribosome profiling data showing the $5^{\prime}$-boundary of ribosomes on the SecM reporter in the strain lacking tmRNA and SmrB. (B) The distribution of FLAG-SmrB with and without mupirocin (MPC) treatment (which induces pauses at lle codons) was determined by fractionation over sucrose gradient and detection with an anti-FLAG antibody. A non-specific band is marked with *. (C) Lysates from cells with and without mupirocin treatment were treated with RNase A, fractionated over sucrose gradients, and the binding of FLAG-SmrB to nuclease resistant disomes was detected with an anti-FLAG antibody. (D) and (E) Low doses of tetracycline induce collisions whereas high doses stall ribosomes without inducing collisions. Following treatment with four different tetracycline concentrations, the distribution of FLAG-SmrB was determined by fractionation over sucrose gradient and detection with an anti-FLAG antibody. (F) and (G) In a new series of reporters, the Crp gene and bleomycin resistant gene are fused with the short SecM motif between them. The Crp gene is trimmed to reduce the number of ribosomes that can be loaded between the start codon and the stall site (the number shown). Reporter mRNA was detected on northern blots using the $3^{\prime}$-probe. An arrow indicates the downstream fragments. Ethidium bromide staining of $16 \mathrm{~S}$ rRNA serves as a loading control. 
peak is modestly but reproducibly higher in the MPC treated samples, consistent with the expectation that there are more ribosome collisions in MPC-treated cells. Although much of the SmrB dissociates from ribosomes under these conditions, moving into the top fractions, we see a strong SmrB band in the nuclease-resistant disome peak in the MPC-treated sample; quantitation of the amount of SmrB bound to various fractions shows strong enrichment for SmrB binding on colliding ribosomes relative to monosomes.

Another strategy to differentiate the effects of stalling and ribosome collisions is to treat cells with antibiotics that target the ribosome and then compare the effects of high doses, which stall all ribosomes quickly, versus lower doses that only stall some ribosomes, allowing others to translate until collisions occur (Fig 4D) (18). In untreated samples SmrB is broadly distributed in sucrose gradients while SmrB is enriched in polysomes deeper in the gradient when $1.25 \mu \mathrm{g} / \mathrm{mL}$ tetracycline is used (Fig 4E). Importantly, the enrichment of SmrB in the polysomes is lost in cells treated with 10 - or 100 -fold higher concentrations of tetracycline.

\section{Ribosome collisions promote SmrB cleavage}

As previously performed in yeast (18), we generated a series of reporters to test whether ribosome collisions are required for mRNA cleavage. In these reporters, different lengths of the crp gene were fused upstream of the short SecM stalling motif, while the downstream ble sequence remains constant (Fig 4F). The reporters are numbered by the distance from the start codon to the stall site (in nt). We anticipated that the closer the short SecM motif is to the $5^{\prime}$-end of the ORF, the less room there is for ribosomes to load onto the mRNA and collide at the stalling motif. Using the 3 '-probe against the reporter mRNA to follow the activity of SmrB, we observe the downstream mRNA fragment characteristic of SmrB cleavage and a strong reduction in fulllength mRNA in the 111, 222, and 423 reporters (Fig 4G). In contrast, the downstream mRNA fragment is reduced in the 51 and 81 reporters and we see much higher levels of full-length mRNA. In the 51 and 81 reporters, only one or two ribosomes can be loaded upstream of the SecM stalled ribosome, respectively, suggesting that multiple collisions may be required to recruit SmrB. In the $\Delta s m r B$ strain, the amount of full-length mRNA is strongly increased in all five reporters and no downstream fragment is observed. These findings show that SmrB activity is triggered by collisions.

The structure of collided ribosomes from B. subtilis and E. coli

Ribosomal collisions in yeast and mammalian cells create a distinct architecture of disomes with new composite interaction surfaces that are recognized by collision sensors (16, 17). To ask whether bacterial ribosomes display a similar behavior, we generated collided ribosomes in cell-free translation systems, translating mRNAs encoding the arrest peptides MifM (32) in B. subtilis extracts and VemP (33) in the commercially available E. coli PURE system. 
Following separation by sucrose density gradient centrifugation, disome and trisome peaks were collected and subjected to structural analysis by cryo-EM.

$3 \mathrm{D}$ reconstruction of the $E$. coli disomes (details in Fig S5) revealed a defined arrangement of two ribosomes (Fig 5A). The leading (stalled) ribosome closely resembles the previously described VemP-stalled 70S in a non-rotated state, carrying a peptidyl-tRNA in the P site, a highly structured nascent peptide chain in the ribosomal tunnel, and an aminoacyl-tRNA in the A site (34). The collided ribosome is found in a mixture of rotated and non-rotated states carrying two tRNAs in the canonical or hybrid state conformation. The stalled ribosome engages the collided ribosome in an intricate interaction mainly involving protein-protein and protein-rRNA interactions between the two juxtaposed small $30 \mathrm{~S}$ subunits. These interactions involve ribosomal proteins uS10, uS2, 16S rRNA helix h16, uS4, and 16S rRNA helices h5 and h17 in the collided ribosome interacting with uS9, uS2, 25S rRNA helix H78, uS11 and bS6, and uL9 of the stalled ribosome, respectively (Fig 5B,C). In addition, the L1 stalk in the large subunit of the stalled ribosome was observed in its "out" conformation forming a new bridge between its rRNA helix $\mathrm{H} 78$ and $16 \mathrm{~S}$ helix $\mathrm{h} 16$ of the collided ribosome (Fig 5C).

Another substantial difference between the conformation of the stalled and collided ribosome is the dramatic rearrangement of the uL9 protein of the large ribosomal subunit. In individual 70S ribosomes, from its binding site on the 50S subunit below the L1 stalk, L9 contacts uS6 and UL2 in the 30S subunit (not shown). While this conformation was also observed in the collided ribosome, the L9 protein of the stalled ribosome flipped out of this position to engage in a novel mode of interaction via its C-terminal domain with the $30 \mathrm{~S}$ subunit of the collided ribosome. This interaction involves rRNA helices $\mathrm{h} 5$ and $\mathrm{h} 17$ of the collided ribosome, thereby effectively forming another bridge between the stalled 50S subunit and the collided 30S subunit (Fig 5C). Although a similar bridging interaction of 19 between individual 70 S ribosomes was observed previously in crystallized $70 S$ ribosomes from $E$. coli and other bacteria $(35,36)$, the L9 binding site on the neighboring $30 \mathrm{~S}$ subunit does not overlap with the site observed here in collided disomes and may be an artifact caused by crystallization conditions.

Notably, the largest protein of the 30S subunit, S1, is missing in the stalled ribosome but present in the collided one (Fig $5 \mathrm{~A}$ ). The arrangement of the interface between the solvent sides of the two 30S subunits results in very limited accessible space and would lead to a steric clash of the S1 protein with the collided ribosome. We conclude that formation of the observed disome architecture requires dissociation of S1 from the stalled ribosome. S1 dissociation may serve as a checkpoint in order to discriminate between short-lived ribosome collisions in productive polysomes and longer lasting stalling events.

When analyzing $E$. coli trisomes, we found that the structural features of the interface between the second and third ribosomes are essentially identical to the interface observed between the stalled and first collided ribosomes (e.g. the L1 and L9 bridges) (Fig 5D). This suggests that during long-lived stalling events, additional collisions can accumulate and yield a multitude 
A
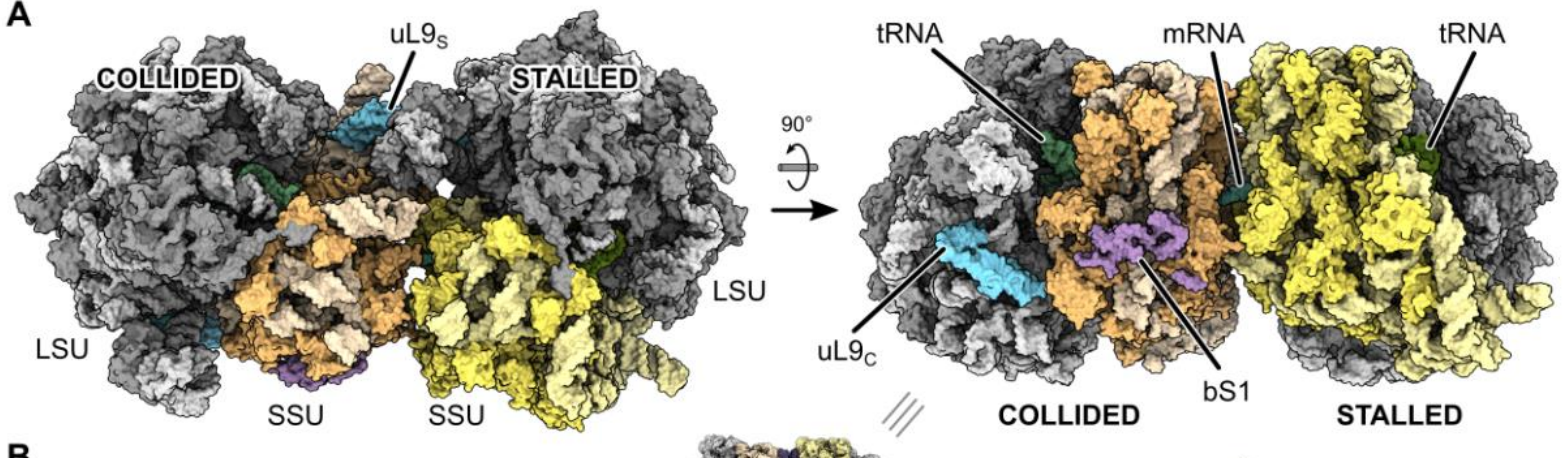

B
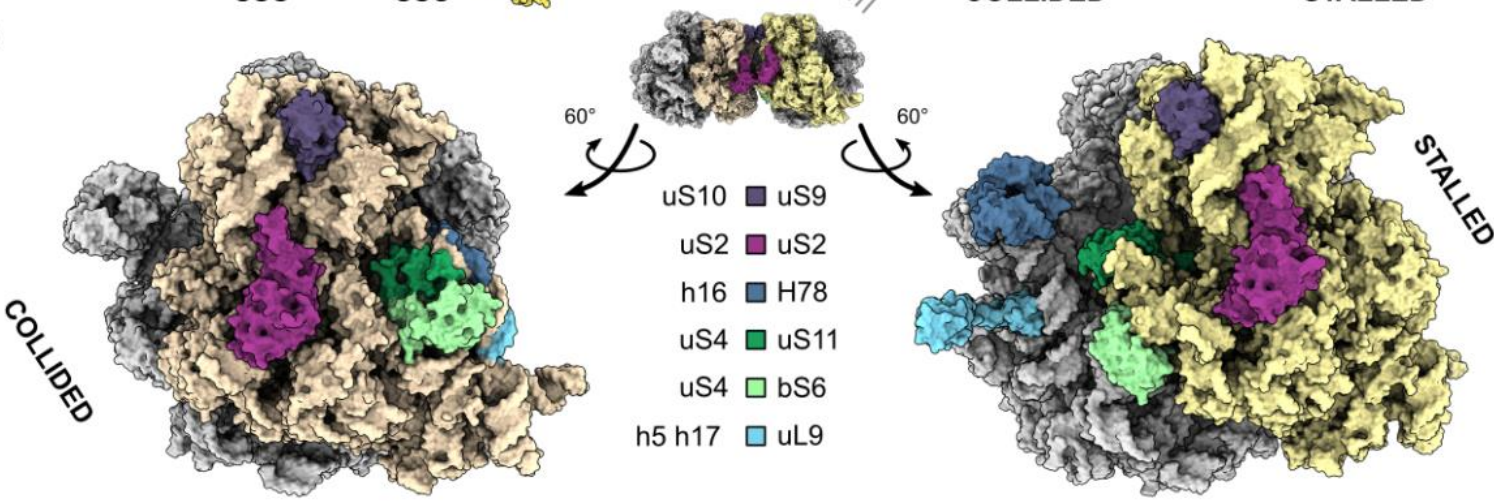

C
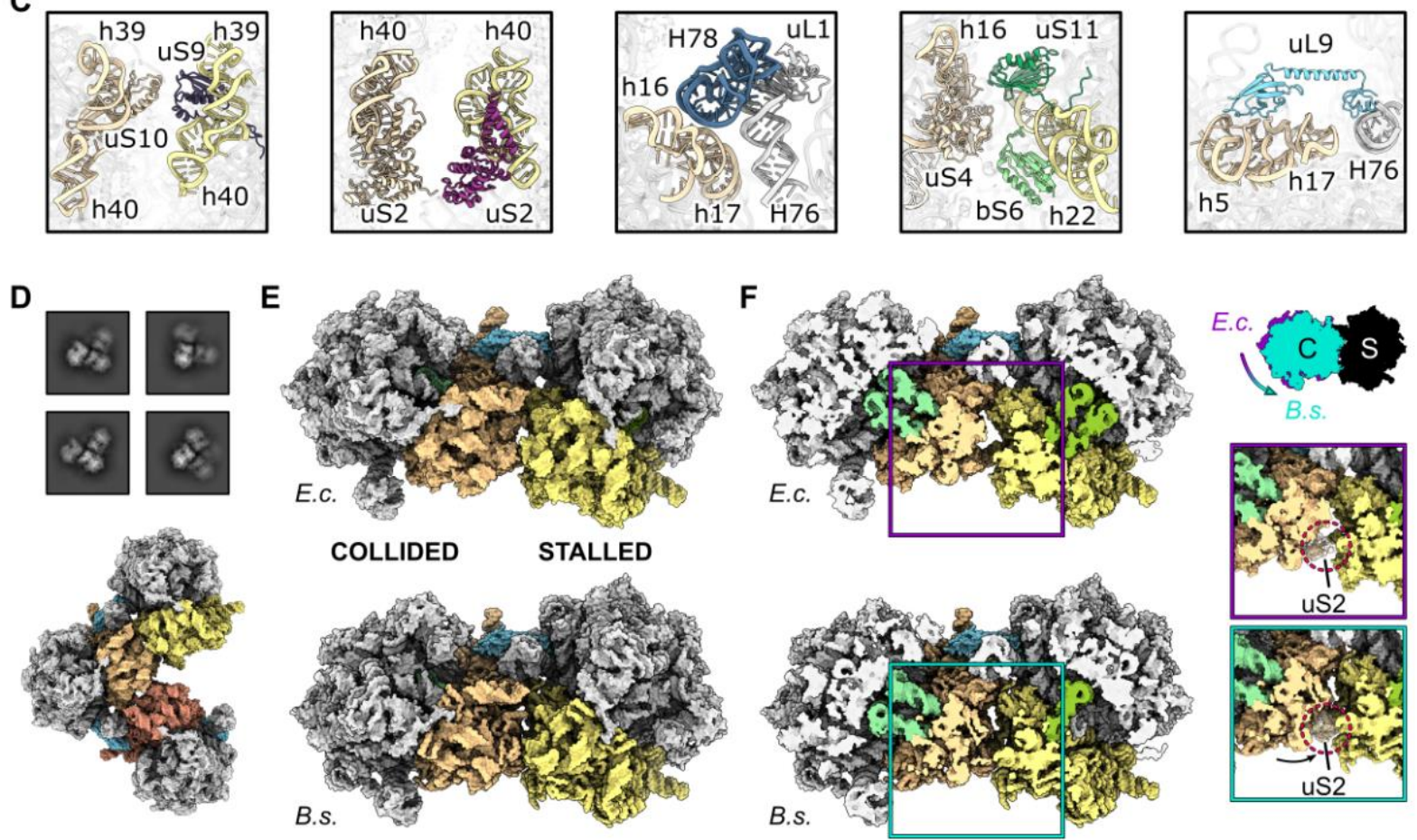

Figure 5. Cryo-EM structure of the $E$. coli disome. (A) Surface representation of the structural model of the E. coli disome. The uL9 proteins from stalled ( $u L 9_{s}$ ) and collided ( $u L 9_{c}$ ) adopt different conformations. (B) Interactions between stalled and collided ribosome at the disome interface. The disome interface is opened up by rotation of the stalled and collided ribosomes and interaction partners are shown in matching colors. (C) Cartoon representation of the individual interactions as they occur at the interface. (D) 2D class averages and cryo-EM structure model of an E. coli trisome. (E and F) Comparison of the E. coli (E.C.) and B. subtilis (B.s.) disomes displaying full and cut views. Note the smaller space between stalled and collided ribosomes in the B.s. disome interface as illustrated by comparing the positions of uS2 proteins in the zoomed view in (F). 
of composite ribosome-ribosome interfaces. This may explain our observation of gradually increasing efficiency of ribosome rescue with longer reporter mRNAs allowing for more ribosomes to collide (Fig 4F,G).

We wondered whether the observed disome formation is unique to $E$. coli and compared our structures to those of the MifM-stalled disomes from the Gram-positive bacterium B. subtilis. We found that ribosome collisions in $B$. subtilis result in disomes adopting an essentially identical conformation as observed in $E$. coli: the overall orientation and interactions of the ribosomes are highly similar, the bridge by the L1 stalk to rRNA helix h16 is formed, and L9 of the stalled ribosome reaches over to the $30 \mathrm{~S}$ subunit of the collided one (Fig 5E). One notable difference is that the 30 s subunits are positioned closer in the B. subtilis disome when compared to E. coli (Fig $5 F)$. This high degree of overall similarity indicates that the observed mode of collided disome formation is likely to be conserved in bacteria, with subtle differences at the disome interface. Notably, the overall architecture of these collided disomes is very different from hibernating, socalled 100 disomes formed under stress conditions (Fig S6) $(37,38)$. We conclude that, similar to eukaryotes, the observed disome (and trisome) architecture of collided ribosomes is a conserved feature in bacteria that can provide a unique interface used by rescue factors such as SmrB to recognize stalled ribosomes.

\section{In vitro cleavage of $M R N A$ and the SmrB-disome structure}

Next, we reconstituted SmrB recruitment to collided disomes and endonucleolytic cleavage of mRNA in vitro. We incubated purified SmrB or the nuclease deficient ALA mutant with purified VemP-stalled disomes and analyzed the reaction products by sucrose density gradient centrifugation (Fig 6A). In a control reaction without SmrB we observed disomes, as expected, as well as some $70 \mathrm{~S}$ ribosomes likely arising from ribosome dissociation from the mRNA and/or background nucleolytic activity. In contrast, after incubation with wild-type but not mutant SmrB, we observed an almost complete loss of the disome signal and a corresponding increase in the $70 \mathrm{~S}$ signal, consistent with cleavage between the collided ribosomes by the endonuclease activity of SmrB.

We reconstituted the nuclease-deficient SmrB mutant with our E. coli VemP-stalled disomes and subjected the complexes to structural analysis by cryo-EM (details in Fig S7). Compared to disomes alone, this reconstruction revealed an extra density between the 30S ribosomal subunits in the immediate vicinity of the mRNA stretching from the mRNA exit site of the stalled ribosome to the mRNA entry of the collided ribosome (Fig 6B). Although the overall resolution of the SmrB-bound disome is $3.3 \AA$, we observed limited local resolution in this region of the map. Therefore, we could only partially build the molecular structure of SmrB and relied on the AF2-driven prediction for rigid body docking of SmrB (39) (Fig S8).

The binding site for SmrB involves both the stalled and the collided ribosome: on the collided ribosome the SMR domain interacts with uS3, uS2 and uS5, whereas on the stalled 
ribosome it binds rRNA helices h40 and h26 as well as ribosomal proteins uS11 and uS21 (Fig 6CE). Notably, in several bacteria the genes encoding uS21 and SMR-domain proteins are tightly linked in conserved operons, suggesting that this interaction might be a conserved aspect of binding of SMR domains to collided ribosomes. The $\mathrm{N}$-terminal extension region of SmrB forms a hook-like structure that wraps around uS2 of the collided ribosome (Fig 6D). Interestingly, the Nterminal alpha-helix contains the --xxxa motif conserved in other SMR-domain proteins in proteobacteria (Fig 2C and Fig S3). We observed this hook-like N-terminus of SmrB on the stalled ribosome as well, although in that position it cannot be connected to the SMR domain between the subunits and thus represents a second copy of SmrB in the complex. We speculate that this $\mathrm{N}$-terminal helix may promote the initial recruitment of SmrB to elongating $70 \mathrm{~S}$ ribosomes and that when collisions occur, the subsequent binding of the SMR domain at the composite binding site between collided ribosomes further stabilizes SmrB binding. In agreement with this idea, a truncated SmrB mutant consisting of only the SMR domain (residues 88-183) does not bind ribosomes (Fig $6 \mathrm{H}$ ). The observed binding mode of SmrB therefore explains how SmrB is specifically recruited to collided disomes (or trisomes).

The active site of SmrB interacts with the bridging mRNA, poised for cleavage in between the individual ribosomes (Fig 6F,G). From the structure, it is difficult to determine the exact mRNA residues to be cleaved, however, and the nuclease-deficient mutant of SmrB may engage in a somewhat different interaction with its mRNA substrate. Nevertheless, the observed positioning indicates that SmrB can execute endonucleolytic cleavage of the mRNA between position -9 and -16 , counting from the first nucleotide in the $A$ site of the stalled ribosome, in agreement with our biochemical data. We speculate that activation of SmrB specifically on collided disomes is a result of precise positioning with respect to its substrate.

\section{Disruption of the SmrB-binding pocket on collided ribosomes}

We asked if disruption of the disome interface or the SmrB binding pocket formed between collided ribosomes would interfere with mRNA cleavage by SmrB. The L9 protein from the stalled ribosome makes contacts with the $30 \mathrm{~S}$ subunit in the collided ribosome (Fig 5C) and strains lacking L9 are known to have high levels of frameshifting $(40,41)$. We found, however, that SmrB still cleaves the CRP reporters in a collision dependent manner in an L9 knockout strain; the reporter mRNA processing is indistinguishable from that seen in wild-type strains (Fig S9). Moreover, fusion of mCherry to the C-terminus of $L 9$ (on the domain that contacts 16S rRNA in the collided ribosome) also has no effect on SmrB activity (Fig S9). These results suggest that this contact between the disomes does not play an essential role in stabilizing the disome interaction. In contrast, we observe that fusion of MBP to the N-terminus of S21 dramatically stabilizes fulllength mRNA in the 111 and 222 CRP reporters compared to the wild-type strain (Fig 6I). To a lesser extent, fusion of GFP to the C-terminus of $\mathrm{S} 6$ also stabilizes the 111 CRP reporter compared to the wild-type strain (Fig $6 \mathrm{I}$ ). These results are consistent with a reduction in SmrB activity and 
bioRxiv preprint doi: https://doi.org/10.1101/2021.08.16.456513; this version posted August 16, 2021. The copyright holder for this preprint (which was not certified by peer review) is the author/funder, who has granted bioRxiv a license to display the preprint in perpetuity. It is made available under aCC-BY-NC-ND 4.0 International license.

stabilization of the reporter mRNA due to a disruption of the SmrB binding pocket formed between the collided ribosomes, validating the structural findings reported here.
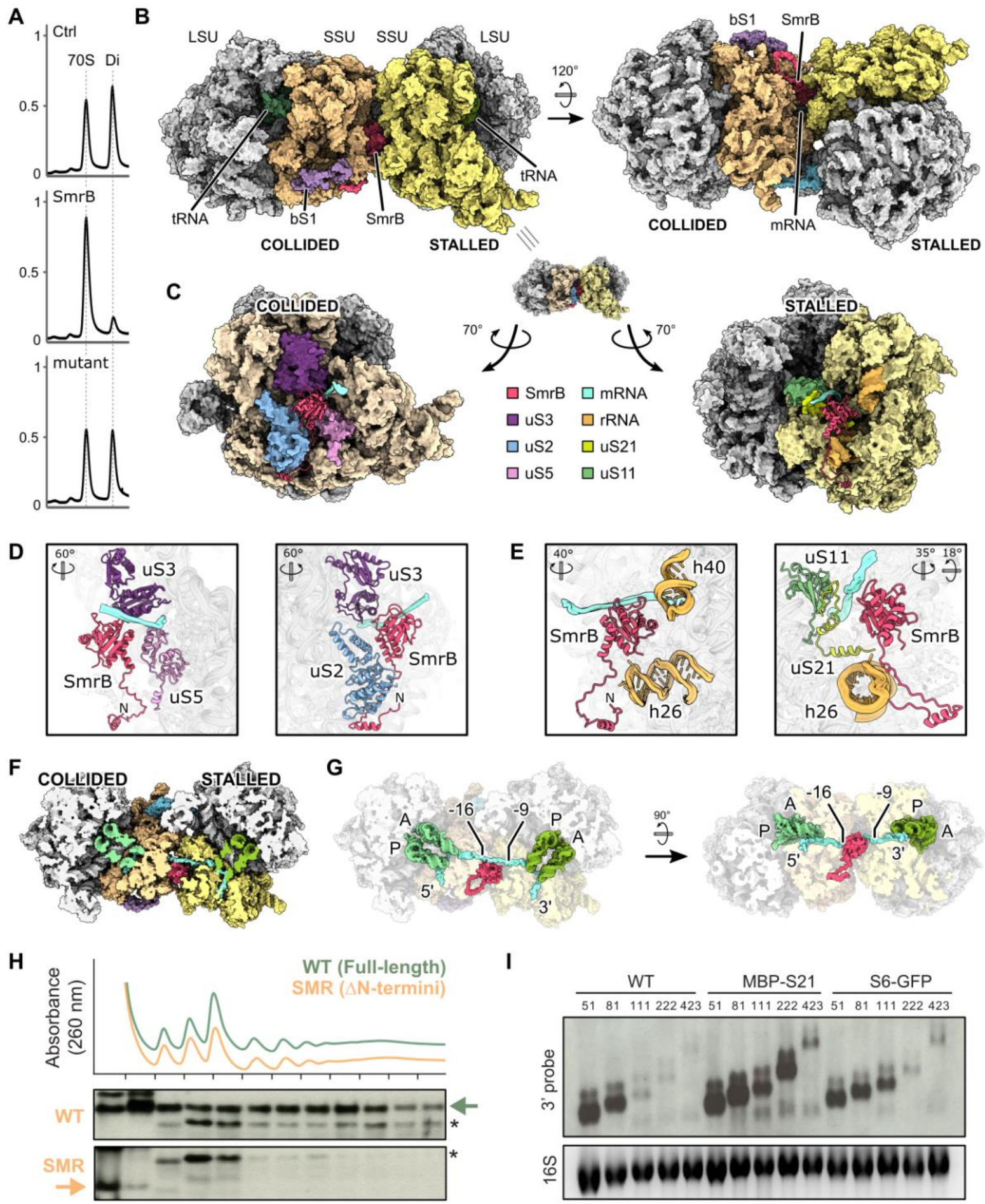

Figure 6. Cryo-EM structure of the SmrB-bound E. coli disome. (A) Disome nuclease assay. Sucrose density gradient profiles of VemP-stalled disomes alone ( $\mathrm{Ctrl}$ ), incubated with SmrB (SmrB) and incubated with nuclease deficient SmrB (mutant), respectively. The Y-axes show the absorption at $260 \mathrm{~nm}$. (B) Surface representation structural model of SmrB bound to the E. coli disome. (C) Interactions between SmrB and the stalled and collided ribosomes at the disome interface. The disome interface is opened up by rotation of the stalled and collided ribosomes and SmrB is shown in cartoon representation. Continued on next page... 
Figure 6. (D and E) Cartoon representation of the individual interactions of SmrB with the collided ribosome in (D) and the stalled ribosome in (E). The orientations of the individual views with respect to $(C)$ are indicated. (F) Cut view of the SmrB bound disome showing the $m R N A$ path. (G) Interaction of SmrB with the mRNA. The disome orientation in the left part of (G) corresponds to the one shown in (F). Approximately 8 nucleotides of the mRNA are exposed at the disome interface, in reach for SmrB cleavage. The nucleotides at the ribosome boundaries are indicated, counting the first nucleotide in the A-site of the stalled ribosome as 0 . (H) The distribution of FLAG-tagged fulllength SmrB and a construct with only the SMR domain (residues 88-183) was determined by fractionation over sucrose gradient and detection with an anti-FLAG antibody. A non-specific band is marked with *. (I) Northern blots using the $3^{\prime}$-probe against the CRP reporters with the short SecM stalling motif in wild-type cells, a strain where MBP is fused to the N-terminus of S21, and a strain where GFP is fused to the C-terminus of S6.

\section{Discussion}

Our findings indicate that ribosome collisions are the critical trigger for ribosome rescue in E. coli: collisions recruit the endonuclease SmrB, triggering mRNA cleavage and rescue of upstream ribosomes by tmRNA. Under normal conditions, SmrB is distributed broadly across sucrose gradients, suggesting that it binds ribosomes generally, scanning for problems. When collisions are induced throughout the transcriptome, SmrB moves deep into the polysome fraction and binds preferentially to nuclease-resistant disomes that are a hallmark of collisions. Importantly, we find that SmrB recruitment is triggered by low doses of tetracycline that promote collisions but not by high doses that promote stalling without collisions. Furthermore, SmrB is unable to cleave mRNA at stalling motifs positioned too close to the 5 '-end of an ORF for sufficient collisions to occur. We conclude that ribosome collisions serve as a signal to recruit ribosome rescue factors in bacteria as well as in eukaryotes.

The importance of collisions is fully validated by our cryo-EM structures of collided ribosomes from both Gram negative $E$. coli cells and Gram positive B. subtilis cells that reveal a specific architecture of closely interacting individual 705 ribosomes. Their architecture is similar to, but distinct from, the collided disome structures previously characterized in eukaryotic yeast and mammalian cells $(16,17)$. In both cases, the interaction between stalled and collided ribosomes primarily involves the two small ribosomal subunits, but also employs contacts between the large subunit of the stalled ribosome and the small subunit of the collided one. However, these contacts are not conserved between the kingdoms: in eukaryotes they are established by $25 \mathrm{~S}$ rRNA helix H31L and ribosomal protein eL27 of the large subunit, whereas in bacteria by the L1 stalk and the ribosomal protein uL9. The collided disome architecture is likely to be conserved in bacteria and, importantly, differs completely from the structure of hibernating disomes in $E$. coli and B. subtilis. This makes the observed collided disomes a valid proxy for sensing ribosome stalling in bacteria.

A role for collisions in ribosome rescue is consistent with previous studies in bacteria. Structural studies of polysomes and the crystal packing interactions in $x$-ray structures show that bacterial ribosomes pack closely together through interactions between their small subunits (35, 42). Moreover, in ribosome profiling studies in E. coli, Subramaniam et al. observed reduced ribosome density downstream of pausing sites due to the removal of stalled ribosomes by tmRNA 
(43); these researchers later argued that reductions in protein output were most consistent with a mathematical model in which collisions are the trigger that recruits rescue factors to remove stalled ribosomes (44). Finally, two recent studies suggest that ribosome collisions influence the level of frameshifting at pause sites in $\operatorname{E}$. coli $(45,46)$.

It is striking that collided ribosomes are recognized in $E$. coli by an SMR-domain protein (SmrB) given that the same domain plays a similar role in yeast (Cue2) (20). Prior to this study, only Rqc2, the factor that promotes CAT-tailing in yeast and has homologs in some bacteria (47, 48), has been shown to mediate ribosome rescue pathways in both bacteria and eukaryotes. Our analysis identified SMR-domain proteins in all but a few bacterial phyla (Fig 2A), suggesting that a role for SMR proteins in ribosome rescue may be widespread in bacteria just as it is in eukaryotes.

In yeast (20), worms (21), and now E. coli, ribosome collisions lead to mRNA cleavage by SMR-domain proteins upstream of the ribosome stalling site, targeting the problematic mRNA for decay. Cleavage leads to the rescue of upstream ribosomes which, no longer impeded by a downstream stalled ribosome, translate to the 3 '-end of the upstream fragment and are released by rescue factors, tmRNA-SmpB in bacteria and Dom34-Hbs1 in yeast. Despite these general similarities, there are also a few apparent differences between the activity of SmrB and the Cue2 protein in yeast. While our RACE and MS data place the SmrB cleavage site at the 5 ' boundary of the stalled ribosome (Fig 3), Cue2 cleavage has been mapped to the A site of the collided ribosome in a wild-type yeast strain and to the $5^{\prime}$ boundary of the stalled ribosome in strains lacking rescue factors Hel2 or $\operatorname{Slh} 1(16,20,49)$. A second difference is that loss of Cue2 alone has little or no effect on the stability of reporter mRNAs in yeast because the processive $5^{\prime}-3^{\prime}$ exonuclease Xrn1 is primarily responsible for their decay (20). In contrast, loss of SmrB leads to a dramatic increase in full-length mRNA in our reporters in E. coli (Fig 2F).

We observe two pathways by which stalled ribosome complexes are resolved (Fig 3I). The main pathway is cleavage at the $5^{\prime}$-boundary of the stalled ribosome by SmrB. In the second pathway, 3'-to-5' exonucleases degrade mRNA until they encounter the stalled ribosome, allowing tmRNA access for rescue to $\operatorname{occur}(30,50)$. E. coli possesses three major 3'-to-5' exonucleases involved in mRNA turnover: RNase II, RNase R, and polynucleotide phosphorylase. Previous studies showed that strains with single knockouts of any of these exonucleases still exhibited trimming of mRNA back to the 3'-boundary of ribosomes stalled at the SecM motif (50); the triple deletion strain is not viable.

The structure of the disome-bound SmrB illustrates how this rescue factor can generally screen translating ribosomes and, in case of problematic events such as mRNA damage by oxidative or alkylating agents (3), is able to specifically recognize the new composite interface formed between the individual collided $70 \mathrm{~S}$ ribosomes. Through its $\mathrm{N}$-terminus, SmrB associates generally with elongating ribosomes for screening, whereas the composite binding site between the adjacent collided 30 S subunits is required to position the SMR domain of SmrB proximal to 
the bridging mRNA for endonucleolytic cleavage. In contrast, eukaryotic SMR-domain proteins frequently contain ubiquitin-binding domains (e.g. CUE, UBA, UIM, and UBL) to bind ribosome proteins ubiquitinylated by E3 ligases such as Hel2. This diversity suggests that recruitment to collided ribosomes can occur through multiple alternate mechanisms.

In this report, we reveal a novel ribosome rescue factor in bacteria, $\mathrm{SmrB}$, that recognizes ribosome collisions and specifically targets stalled ribosomes for mRNA decay and ribosome rescue. Bacteria and eukaryotes both rely on collisions to sense ribosome stalling and SMRdomain proteins to cleave the mRNA so that upstream stalled ribosomes can then be rescued by factors known to act on truncated mRNAs. These common features substantiate the universal significance of ribosome collisions and endonucleolytic cleavage in ribosome rescue. We anticipate the bacterial system to be as complex as the eukaryotic counterpart that enables recognition of stalled ribosomes, triggering RNA and proteolytic processing, and rescuing ribosomes. Our present work provides the initial scaffold for additional studies to fully appreciate this phenomenon.

Acknowledgements: The authors thank Suparna Sanyal for sharing E. coli strains QC101 and QC901, Haiping Hao at the JHMI Transcriptomics and Deep Sequencing Core for assistance with high-throughput sequencing, Bob Cole and Tatiana Boronina at JHMI in the Mass Spectrometry and Proteomics Facility, Joanna Musial for assistance during protein purifications, C. Ungewickell and S. Rieder for technical assistance, and L. Kater and K. Best for support with the pre-processing pipeline of cryo-EM data. Funding: This work was supported by NIH grant GM136960 (ARB), HHMI (RG), the Intramural Research Program of the National Library of Medicine at the NIH (AMB and LA), and by German Research Council (TRR174) (RB). HK is supported by a DFG fellowship through the Graduate School of Quantitative Bioscience Munich (QBM). Author contributions: K.S. performed the genetic screen, the analyses of the nanoLuc-ble reporters, and sucrose gradients. A.C. analyzed the CRP reporters and prepared samples for the MS experiments. M.B. and L.A. performed the phylogenetic analyses. H.K. performed in vitro translation and in vitro nuclease assays and prepared samples for cryo-EM analysis. O.B. and R.Bu. collected cryo-EM data. H.K. processed the cryo-EM data and R.Bu. prepared molecular models. H.K., R.Bu. and R.Be. analyzed and interpreted the structures and R.Bu. prepared structural figures. A.B., R.G., and R.Be. supervised the project. Competing Interests: The authors declare that there are no competing interests. Data and materials availability: The ribosome profiling data are available at the GEO with accession number GSE179691. Cryo-EM volumes and molecular models have been deposited at the Electron Microscopy Data Bank and Protein Data Bank with accession codes EMD-XXXX (E. coli maps) and PDB-YYYY (stalled 70S) PDB-ZZZZ (collided 70S-SmrB), EMDYYYY (B. subtilis maps). 
bioRxiv preprint doi: https://doi.org/10.1101/2021.08.16.456513; this version posted August 16, 2021. The copyright holder for this preprint

(which was not certified by peer review) is the author/funder, who has granted bioRxiv a license to display the preprint in perpetuity. It is made available under aCC-BY-NC-ND 4.0 International license.

\section{Supplementary Materials}

Materials and Methods

Table S1

Fig S1 - S9

References (51-74) 


\section{References:}

1. S. D. Moore, R. T. Sauer, Ribosome rescue: tmRNA tagging activity and capacity in Escherichia coli. Molecular Microbiology 58, 456-466 (2005).

2. L. L. Yan, H. S. Zaher, How do cells cope with RNA damage and its consequences? The Journal of Biological Chemistry 294, 15158-15171 (2019).

3. E. N. Thomas, K. Q. Kim, E. P. McHugh, T. Marcinkiewicz, H. S. Zaher, Alkylative damage of mRNA leads to ribosome stalling and rescue by trans translation in bacteria. elife 9, e61984 (2020).

4. E. D. Roche, R. T. Sauer, SsrA-mediated peptide tagging caused by rare codons and tRNA scarcity. The EMBO journal 18, 4579-4589 (1999).

5. T. Sunohara, K. Jojima, H. Tagami, T. Inada, H. Aiba, Ribosome stalling during translation elongation induces cleavage of mRNA being translated in Escherichia coli. The Journal of Biological Chemistry 279, 15368-15375 (2004).

6. C. S. Hayes, B. Bose, R. T. Sauer, Proline residues at the $\mathrm{C}$ terminus of nascent chains induce SsrA tagging during translation termination. The Journal of Biological Chemistry 277, 33825-33832 (2002).

7. C. Müller, C. Crowe-McAuliffe, D. N. Wilson, Ribosome Rescue Pathways in Bacteria. Frontiers in Microbiology 12, 652980 (2021).

8. F. Garza-Sánchez, R. E. Schaub, B. D. Janssen, C. S. Hayes, tmRNA regulates synthesis of the ArfA ribosome rescue factor. Molecular Microbiology 80, 1204-1219 (2011).

9. K. C. Keiler, P. R. Waller, R. T. Sauer, Role of a peptide tagging system in degradation of proteins synthesized from damaged messenger RNA. Science (New York, N.Y.) 271, 990993 (1996).

10. Y. Chadani, K. Ito, K. Kutsukake, T. Abo, ArfA recruits release factor 2 to rescue stalled ribosomes by peptidyl-tRNA hydrolysis in Escherichia coli. Molecular Microbiology 86, 3750 (2012).

11. N. Ivanova, M. Y. Pavlov, B. Felden, M. Ehrenberg, Ribosome rescue by tmRNA requires truncated mRNAs. Journal of Molecular Biology 338, 33-41 (2004).

12. C. Neubauer, R. Gillet, A. C. Kelley, V. Ramakrishnan, Decoding in the absence of a codon by tmRNA and SmpB in the ribosome. Science (New York, N.Y.) 335, 1366-1369 (2012).

13. D. Kurita, Y. Chadani, A. Muto, T. Abo, H. Himeno, ArfA recognizes the lack of mRNA in the mRNA channel after RF2 binding for ribosome rescue. Nucleic Acids Research 42, 1333913352 (2014).

14. C. S. Hayes, R. T. Sauer, Cleavage of the A site mRNA codon during ribosome pausing provides a mechanism for translational quality control. Molecular Cell 12, 903-911 (2003).

15. O. Brandman et al., A ribosome-bound quality control complex triggers degradation of nascent peptides and signals translation stress. Cell 151, 1042-1054 (2012). 
16. K. Ikeuchi et al., Collided ribosomes form a unique structural interface to induce Hel2driven quality control pathways. The EMBO journal 38, e100276 (2019).

17. S. Juszkiewicz et al., ZNF598 Is a Quality Control Sensor of Collided Ribosomes. Molecular Cell 72, 469-481.e467 (2018).

18. C. L. Simms, L. L. Yan, H. S. Zaher, Ribosome Collision Is Critical for Quality Control during No-Go Decay. Molecular Cell 68, 361-373.e365 (2017).

19. W. Zhou et al., PPR-SMR protein SOT1 has RNA endonuclease activity. Proceedings of the National Academy of Sciences of the United States of America 114, E1554-E1563 (2017).

20. K. N. D'Orazio et al., The endonuclease Cue2 cleaves mRNAs at stalled ribosomes during No Go Decay. eLife 8, e49117 (2019).

21. M. L. Glover et al., NONU-1 Encodes a Conserved Endonuclease Required for mRNA Translation Surveillance. Cell Reports 30, 4321-4331.e4324 (2020).

22. T. van Opijnen, K. L. Bodi, A. Camilli, Tn-seq: high-throughput parallel sequencing for fitness and genetic interaction studies in microorganisms. Nature Methods 6, 767-772 (2009).

23. G. C. Langridge et al., Simultaneous assay of every Salmonella Typhi gene using one million transposon mutants. Genome Research 19, 2308-2316 (2009).

24. D. Bhandari, K. Guha, N. Bhaduri, P. Saha, Ubiquitination of mRNA cycling sequence binding protein from Leishmania donovani (LdCSBP) modulates the RNA endonuclease activity of its Smr domain. Febs Lett 585, 809-813 (2011).

25. W. J. Wu et al., SOT1, a pentatricopeptide repeat protein with a small MutS-related domain, is required for correct processing of plastid 23S-4.5S rRNA precursors in Arabidopsis thaliana. Plant J 85, 607-621 (2016).

26. S. Liu, J. Melonek, L. M. Boykin, I. Small, K. A. Howell, PPR-SMRs: ancient proteins with enigmatic functions. RNA biology 10, 1501-1510 (2013).

27. D. H. Bechhofer, M. P. Deutscher, Bacterial ribonucleases and their roles in RNA metabolism. Critical Reviews in Biochemistry and Molecular Biology 54, 242-300 (2019).

28. F. Mohammad, R. Green, A. R. Buskirk, A systematically-revised ribosome profiling method for bacteria reveals pauses at single-codon resolution. elife 8, e42591 (2019).

29. H. Muto, H. Nakatogawa, K. Ito, Genetically encoded but nonpolypeptide prolyl-tRNA functions in the A site for SecM-mediated ribosomal stall. Molecular Cell 22, 545-552 (2006).

30. B. D. Janssen, F. Garza-Sánchez, C. S. Hayes, A-site mRNA cleavage is not required for tmRNA-mediated ssrA-peptide tagging. PloS One 8, e81319 (2013).

31. J. Hughes, G. Mellows, Inhibition of isoleucyl-transfer ribonucleic acid synthetase in Escherichia coli by pseudomonic acid. The Biochemical Journal 176, 305-318 (1978).

32. S. Chiba, A. Lamsa, K. Pogliano, A ribosome-nascent chain sensor of membrane protein biogenesis in Bacillus subtilis. The EMBO journal 28, 3461-3475 (2009). 
33. E. Ishii et al., Nascent chain-monitored remodeling of the Sec machinery for salinity adaptation of marine bacteria. Proceedings of the National Academy of Sciences of the United States of America 112, E5513-5522 (2015).

34. T. Su et al., The force-sensing peptide VemP employs extreme compaction and secondary structure formation to induce ribosomal stalling. eLife 6, e25642 (2017).

35. B. S. Schuwirth et al., Structures of the bacterial ribosome at $3.5 \mathrm{~A}$ resolution. Science (New York, N.Y.) 310, 827-834 (2005).

36. M. Selmer, Y. G. Gao, A. Weixlbaumer, V. Ramakrishnan, Ribosome engineering to promote new crystal forms. Acta Crystallogr D 68, 578-583 (2012).

37. B. Beckert et al., Structure of the Bacillus subtilis hibernating $100 \mathrm{~S}$ ribosome reveals the basis for 70S dimerization. Embo Journal 36, 2061-2072 (2017).

38. B. Beckert et al., Structure of a hibernating $100 \mathrm{~S}$ ribosome reveals an inactive conformation of the ribosomal protein S1. Nat Microbio/ 3, 1115-+ (2018).

39. J. Jumper et al., Highly accurate protein structure prediction with AlphaFold. Nature, (2021).

40. K. L. Herbst, L. M. Nichols, R. F. Gesteland, R. B. Weiss, A mutation in ribosomal protein L9 affects ribosomal hopping during translation of gene 60 from bacteriophage T4. Proceedings of the National Academy of Sciences of the United States of America 91, 12525-12529 (1994).

41. R. Leipuviene, G. R. Björk, Alterations in the two globular domains or in the connecting alpha-helix of bacterial ribosomal protein 19 induces +1 frameshifts. Journal of Bacteriology 189, 7024-7031 (2007).

42. F. Brandt et al., The native 3D organization of bacterial polysomes. Cell 136, 261-271 (2009).

43. A. R. Subramaniam, B. M. Zid, E. K. O'Shea, An integrated approach reveals regulatory controls on bacterial translation elongation. Cell 159, 1200-1211 (2014).

44. M. A. Ferrin, A. R. Subramaniam, Kinetic modeling predicts a stimulatory role for ribosome collisions at elongation stall sites in bacteria. eLife 6, e23629 (2017).

45. C. L. Simms, L. L. Yan, J. K. Qiu, H. S. Zaher, Ribosome Collisions Result in +1 Frameshifting in the Absence of No-Go Decay. Cell Reports 28, 1679-1689.e1674 (2019).

46. A. M. Smith, M. S. Costello, A. H. Kettring, R. J. Wingo, S. D. Moore, Ribosome collisions alter frameshifting at translational reprogramming motifs in bacterial mRNAs. Proceedings of the National Academy of Sciences of the United States of America 116, 21769-21779 (2019).

47. P. S. Shen et al., Protein synthesis. Rqc2p and 60S ribosomal subunits mediate mRNAindependent elongation of nascent chains. Science (New York, N.Y.) 347, 75-78 (2015).

48. I. Lytvynenko et al., Alanine Tails Signal Proteolysis in Bacterial Ribosome-Associated Quality Control. Cell 178, 76-90.e22 (2019). 
49. A. Navickas et al., No-Go Decay mRNA cleavage in the ribosome exit tunnel produces 5'$\mathrm{OH}$ ends phosphorylated by Trl1. Nature Communications 11, 122 (2020).

50. F. Garza-Sánchez, B. D. Janssen, C. S. Hayes, Prolyl-tRNA(Pro) in the A-site of SecMarrested ribosomes inhibits the recruitment of transfer-messenger RNA. The Journal of Biological Chemistry 281, 34258-34268 (2006). 